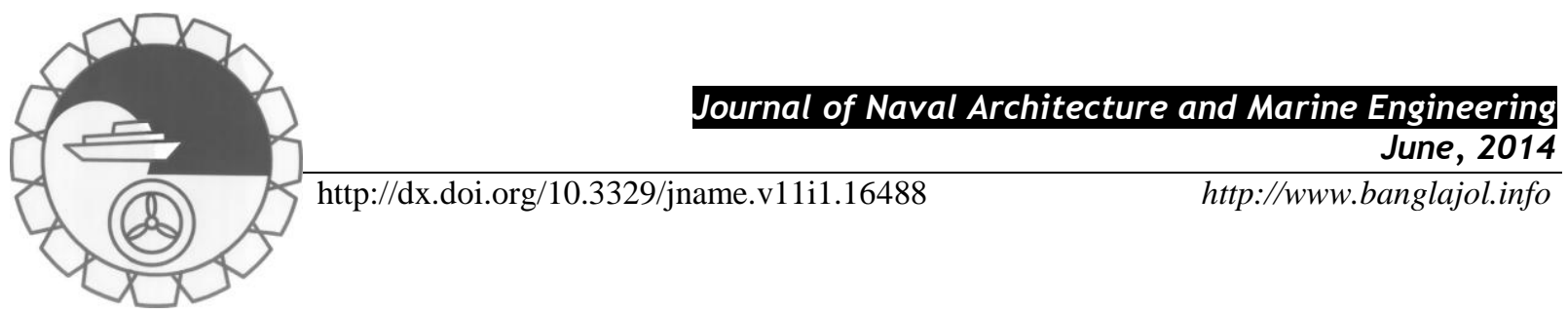

\title{
EFFECT OF VARIABLE THERMAL CONDUCTIVITY ON CONVECTIVE HEAT AND MASS TRANSFER OVER A VERTICAL PLATE IN A ROTATING SYSTEM WITH VARIABLE POROSITY REGIME
}

\author{
R. Bhuvanavijaya ${ }^{1}$ and B. Mallikarjuna ${ }^{2}$
}

${ }^{1}$ Department of Mathematics, JNT University College of Engineering, Anantapur, Ananthapuramu, AndhraPradesh -515002, India, Email: bhuvanarachamalla@gmail.com

${ }^{2}$ Department of Mathematics, JNT University College of Engineering, Anantapur, Ananthapuramu, AndhraPradesh -515002, India, Email: mallikarjuna.jntua@gmail.com

\begin{abstract}
:
Mathematical model has been presented to investigate the effect of variable thermal conductivity on Darcy mixed convective heat and mass transfer flow past a vertical plate in a rotating system by taking into account of variable porosity regime and chemical reaction parameter. The governing boundary layer equations for the flow, energy and species are transformed into system of ordinary differential equations using similarity transformations and then solved numerically by employing Fourth order Runge-Kutta method with shooting technique for various values of physical parameters. The effects of variable thermal conductivity, chemical reaction parameter and variable porosity regime on the fluid velocity, temperature and concentration, local skin friction and rate of heat and mass transfer distribution in the regime are presented graphically and analyzed in detail. We compare the results with previously published work and good agreement is obtained.
\end{abstract}

Keywords: Heat and mass transfer, variable porosity, variable thermal conductivity, chemical reaction

\section{NOMENCLATURE}

C Fluid concentration

$c_{p} \quad$ Specific heat at constant pressure

$c_{s} \quad$ Concentration susceptibility

$C_{w} \quad$ Concentration at the wall

$C_{\infty} \quad$ Concentration of the fluid in free stream

$D_{m} \quad$ Mass diffusivity

Du Dufour parameter

$f_{w} \quad$ Dimensionless suction velocity

$g \quad$ Acceleration due to gravity

$g_{c} \quad$ Mass buoyancy parameter

Gm Modified Grashof number

Gr Grashof number

$g_{s} \quad$ Temperature buoyancy parameter

$K \quad$ Permeability of the porous medium

$k_{T} \quad$ Thermal diffusion ratio

$k_{l} \quad$ Chemical reaction rate

Pr Prandtl number

$\varepsilon \quad$ Porosity of the porous medium

$\begin{array}{ll}R & \text { Rotational Parameter } \\ R e & \text { Reynolds number } \\ S r & \text { Soret number } \\ T & \text { Temperature of the fluid } \\ T_{w} & \text { The plate temperature } \\ T_{\infty} & \text { Fluid temperature in free stream } \\ T_{\infty} & \text { Fluid asymptotic temperature } \\ U_{\infty} & \text { Free steam velocity } \\ u & \text { Velocity component in x-direction } \\ v & \text { Velocity component in y-direction } \\ w & \text { Velocity component in z-direction }\end{array}$

Greek symbols

$\alpha \quad$ Thermal conductivity

$\alpha_{o} \quad$ Thermal diffusivity of the fluid

$\beta \quad$ Thermal conductivity parameter

$\beta_{t} \quad$ Coefficient of thermal expansion

$\beta_{c} \quad$ Coefficient of concentration expansion

$\eta \quad$ Similarity variable 


$\begin{array}{llll}\gamma & \text { Chemical reaction parameter } & v & \text { Kinematic Viscosity } \\ \phi & \text { Non-dimensional concentration } & \psi & \text { Stream function } \\ \theta & \text { Non-dimensional temperature } & \Omega & \text { Angular Velocity about the y-axis }\end{array}$

\section{Introduction}

The study of rotating flow and heat transfer has received wide interest in modern fluid dynamics research with applications in geophysics and planetary sciences. Anwar Beg et. al. (2005) have studied convection heat transfer in a rotating fluid in a thermally stratified high-porosity medium, they employed numerical finite difference method to solve the boundary layer equations. Chamkha et. al (2006) investigated double diffusive convection of a rotating fluid over a surface embedded in a thermally stratified high-porosity medium. Mbeledogu and Ogulu (2007) analyzed heat and mass transfer of an unsteady MHD natural convection flow of a rotating fluid past a vertical flat plate in the presence of radiative heat transfer. Bhargava et. al. (2008) studied transient couette flow in a rotating non-Darcian porous medium parallel plate configuration: network simulation method solutions. Salah et. al (2011) investigated new exact solutions for MHD transient rotating flow of a second-grade fluid in a porous medium. Chauhan and Agarwal (2012) have been studied effects of hall current on MHD couette flow in a channel partially filled with a porous medium in a rotating system.

Most of the papers treated permeability of the porous medium as constant. Schwartz and Smith (1953) investigated and reported porosity is not constant but varies from the wall to the interior due to variation in permeability. At first Vafai and Tien (1981) studied boundary and inertia effects on flow and heat transfer in a porous media of constant and variable porosity. They have observed that the great influence of the variation of porosity and permeability take place in velocity and heat transfer. Pal and Mondal (2009) considered radiation effects on combined convection over a vertical flat plate embedded in a porous medium of variable porosity. Pal (2010) investigated Magnetohydrodynamic non-Darcy mixed convection heat transfer from a vertical heated plate embedded in a porous medium with variable porosity. Prasad et.al (2012) studied thermal radiation effects on magnetohydrodynamic free convection heat and mass transfer from a sphere in a variable porosity regime.

All the above mentioned studies confined their discussions by assuming constant fluid properties. However, it is well known that the physical properties of fluid may vary significantly with temperature. Seddeek and Salama (2007) have considered the effect of temperature dependent viscosity and thermal conductivity on unsteady MHD convective heat transfer past a semi-infinite vertical porous moving plate with variable suction. Mukhopadhyay (2009) analyzed unsteady boundary layer flow and heat transfer past a porous stretching sheet in presence of variable viscosity and thermal diffusivity. Non-Darcy free convection flow over a horizontal cylinder in a saturated porous medium with variable propertied is investigated by Hassanien and Rashed (2011). Vajravelu et.al (2013) studied unsteady convective boundary layer flow of a viscous fluid at a vertical surface with variable fluid properties.

Motivated by the above literature and application, the present study explores the effects of variable porosity, variable thermal conductivity and chemical reaction on mixed convective boundary layer flow, heat and mass transfer over a vertical plate embedded in a porous medium in a rotating system. In the present article we study the influence of the governing physical parameters, variable porosity, variable thermal conductivity and chemical reaction parameter, on heat and mass transfer using a Runge-Kutta method with shooting technique (see Narayana et.al (2013) and Srinivasacharya and Swamy Reddy (2013)). To the best of my knowledge such a study is not appeared in the scientific literature.

\section{Mathematical Formulation}

Let us consider steady, two-dimensional, laminar, incompressible, combined heat and mass transfer flow from a permeable vertical plate embedded in a variable porosity medium in rotating system. Fig. 1 illustrates the physical model and coordinate system. Here $\mathrm{x}$ is measured along the vertical plate, $\mathrm{y}$ is measured normal to the surface, respectively. It is assumed that the free stream velocity $U_{\infty}$, parallel to the vertical plate is constant. The plate is maintained at uniform temperature $T_{w}$ and uniform concentration $C_{w}$ which are higher than $T_{\infty}$ and $C_{\infty}$ respectively, Where $T_{\infty}$ and $C_{\infty}$ temperature and concentration of the fluid far away from the surface of the plate. Initially the plate is at rest, after that the whole system is allowed to rotate with a constant 
angular velocity $\Omega$ about the y-axis. In light of the above assumptions and under Bousinessq approximations, the boundary layer equations for mass, momentum, energy and concentration can be written as follows:

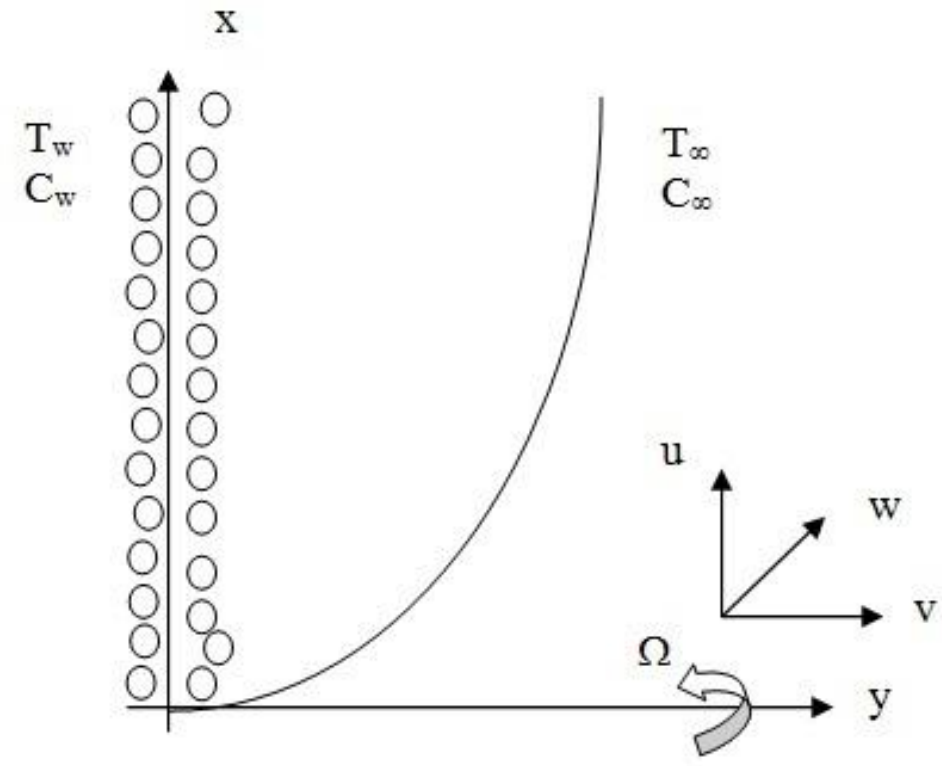

Fig. 1: Physical model and coordinate system

$$
\begin{aligned}
& \frac{\partial u}{\partial x}+\frac{\partial v}{\partial y}=0 \\
& \varepsilon^{-2}\left(u \frac{\partial u}{\partial x}+v \frac{\partial u}{\partial y}-2 \Omega w\right)=\varepsilon^{-1} v \frac{\partial^{2} u}{\partial y^{2}}-\frac{v}{K} u+g \beta_{t}\left(T-T_{\infty}\right)+g \beta_{c}\left(C-C_{\infty}\right) \\
& \varepsilon^{-2}\left(u \frac{\partial w}{\partial x}+v \frac{\partial w}{\partial y}+2 \Omega u\right)=\varepsilon^{-1} v \frac{\partial^{2} w}{\partial y^{2}}-\frac{v}{K} w \\
& u \frac{\partial T}{\partial x}+v \frac{\partial T}{\partial y}=\frac{\partial}{\partial y}\left(\alpha \frac{\partial T}{\partial y}\right)+\frac{D_{m} k_{T}}{c_{s} c_{p}} \frac{\partial^{2} C}{\partial y^{2}} \\
& u \frac{\partial C}{\partial x}+v \frac{\partial C}{\partial y}=D_{m} \frac{\partial^{2} C}{\partial y^{2}}+\frac{D_{m} k_{T}}{T_{m}} \frac{\partial^{2} T}{\partial y^{2}}-k_{l}\left(C-C_{\infty}\right)
\end{aligned}
$$

The boundary conditions are defined as follows:

$$
\begin{aligned}
& u=0, \quad v= \pm v(x), \quad T=T_{w}, \quad C=C_{w} \quad \text { at } \quad y=0 \\
& u \rightarrow U_{\infty}, \quad T \rightarrow T_{\infty}, \quad C \rightarrow C_{\infty} \quad \text { as } \quad y \rightarrow \infty
\end{aligned}
$$

Where $\mathrm{u}, \mathrm{v}$ and $\mathrm{w}$ denotes velocity components in $\mathrm{x}, \mathrm{y}$ and $\mathrm{z}$ directions, respectively, $\mathrm{K}$ is the permeability of the porous medium, $v$ is the kinematic viscosity, $\varepsilon$ is the porosity, $\beta_{\mathrm{t}}$ and $\beta_{\mathrm{c}}$ are the coefficients of thermal expansion and concentration expansions, respectively, $\Omega$ is the angular velocity about the $\mathrm{y}$-axis, $\mathrm{T}$ and $\mathrm{C}$ are temperature and concentration respectively. $\alpha$ and $\mathrm{D}_{\mathrm{m}}$ are thermal and mass diffusivities respectively, $\mathrm{k}_{\mathrm{T}}$ is the thermal diffusion ratio, $\mathrm{c}_{\mathrm{p}}$ is the specific heat at constant pressure, $\mathrm{c}_{\mathrm{s}}$ is the concentration susceptibility, $\mathrm{T}_{\mathrm{m}}$ is the mean fluid temperature and $\mathrm{k}_{\mathrm{l}}$ is the chemical reaction rate and $\mathrm{g}$ is the acceleration due to gravity.

The fluid properties are assumed to be isotropic and constant, except for the fluid thermal conductivity $\alpha$. We assume that the fluid thermal conductivity is to be varying as a linear function of the temperature in the form (Seddeek and Salem (2007)):

$$
\alpha=\alpha_{o}\left(1+E\left(T-T_{\infty}\right)\right)
$$

Where $\mathrm{E}$ is a constant depending on the nature of the fluid and $\alpha_{o}$ is the thermal diffusivity at the surface temperature $\mathrm{T}_{\mathrm{w}}$. In non-dimensional form 


$$
\alpha=\alpha_{o}(1+\beta \theta)
$$

where $\beta=E\left(T_{w}-T_{\infty}\right)$ is the thermal conductivity parameter. The range of the $\beta$ can be taken as $-0.1 \leq \beta \leq 0$ for lubrication oils, $0 \leq \beta \leq 0.12$ for water.

Using Eq. (7), Eq. (4) becomes

$u \frac{\partial T}{\partial x}+v \frac{\partial T}{\partial y}=\alpha_{o} \frac{\partial}{\partial y}\left((1+\beta \theta) \frac{\partial T}{\partial y}\right)+\frac{D_{m} k_{T}}{c_{s} c_{p}} \frac{\partial^{2} C}{\partial y^{2}}$

We now introduce the following similarity variables

$\eta=y \sqrt{\frac{U_{\infty}}{v x}} ; \quad \psi=\sqrt{v x U_{\infty}} f(\eta) ; \quad \theta(\eta)=\frac{T-T_{\infty}}{T_{w}-T_{\infty}} ; \quad \phi(\eta)=\frac{C-C_{\infty}}{C_{w}-C_{\infty}} ; g=\frac{w}{U_{\infty}}$

where $\psi$ is the stream function such that $(u, v)=\left(\frac{\partial \psi}{\partial y},-\frac{\partial \psi}{\partial x}\right)$ and $\eta$ is the similarity variable.

Using Eq. (9), Eqs. (1)-(3), (5) and (8) become

$\varepsilon^{-1} f^{\prime \prime \prime}+\varepsilon^{-2}\left(\frac{1}{2} f f^{\prime \prime}+R g\right)+g_{s} \theta+g_{c} \phi-K f^{\prime}=0$

$\varepsilon^{-1} g^{\prime \prime}+\varepsilon^{-2}\left(\frac{1}{2} f g^{\prime}-R f^{\prime}\right)-K g=0$

$(1+\beta \theta) \theta^{\prime \prime}+\beta\left(\theta^{\prime}\right)^{2}+\frac{1}{2} \operatorname{Pr} f \theta^{\prime}+\operatorname{Pr} D u \phi^{\prime \prime}=0$

$\phi^{\prime \prime}+\frac{1}{2} S c f \phi^{\prime}-S c \gamma \phi+S c S r \theta^{\prime \prime}=0$

The associated boundary conditions are

$$
\begin{aligned}
& f=f_{w}, \quad f^{\prime}=0, \quad g=0, \quad \theta=1, \quad \phi=1 \quad \text { at } \quad \eta \rightarrow 0 \\
& f^{\prime} \rightarrow 1, \quad g \rightarrow 0, \quad \theta \rightarrow 0, \quad \phi \rightarrow 0 \quad \text { as } \quad \eta \rightarrow \infty
\end{aligned}
$$

where $R=\frac{4 \Omega x}{U_{\infty}}$ is the rotational parameter, $g_{s}=\frac{G r}{\mathrm{Re}^{2}}$ is the temperature buoyancy parameter,

$g_{c}=\frac{G m}{\mathrm{Re}^{2}}$ is the mass buoyancy parameter, $G r=\frac{g \beta_{t}\left(T_{w}-T_{\infty}\right) x^{3}}{v^{2}}$ is the Grashof number,

$G m=\frac{g \beta_{c}\left(C_{w}-C_{\infty}\right) x^{3}}{v^{2}}$ is the modified Grashof number, $K=\frac{2 v x}{k U_{\infty}}$ is the Darcy number, $\operatorname{Pr}=\frac{v}{\alpha_{o}}$ is the Prandtl number, $S c=\frac{v}{D}$ is the Schmidt number, $D u=\frac{D_{m} k_{T}}{c_{s} c_{p}} \frac{\left(C_{w}-C_{\infty}\right)}{\left(T_{w}-T_{\infty}\right)}$ is the Dufour parameter, $S r=\frac{D_{m} k_{T}}{v T_{m}} \frac{\left(T_{w}-T_{\infty}\right)}{\left(C_{w}-C_{\infty}\right)}$ is the Soret number, $\gamma=\frac{k_{l}\left(C_{w}-C_{\infty}\right) 2 x}{U_{\infty}}$ is the chemical reaction parameter and $f_{w}=\frac{2 x v(x)}{v} \operatorname{Re}^{-1 / 2}$ is the dimensionless suction velocity.

\section{Numerical Method of Solution}

The a set of nonlinear non-homogeneous differential equation (10)-(13) with corresponding boundary conditions (14) are solved numerically using a shooting technique along with fourth order Runge-Kutta integration. In this method, Equations (10)-(13) are converted into the following system of linear differential equations of first order 


$$
\begin{aligned}
& f^{\prime}=L, f^{\prime \prime}=M, g^{\prime}=N, \\
& \theta^{\prime}=P, \varphi^{\prime}=Q \\
& f^{\prime \prime \prime}=-\varepsilon\left(\varepsilon^{-2}\left(\frac{1}{2} f M+R g\right)-K L+g_{s} \theta+g_{c} \phi\right) \\
& g^{\prime \prime}=-\varepsilon\left(\varepsilon^{-2}\left(\frac{1}{2} f N-R L\right)-K g\right) \\
& \theta^{\prime \prime}=\frac{-1}{(1+\beta \theta)-\operatorname{Pr} D u S c S r}\left[\beta(P)^{2}+\frac{1}{2} \operatorname{Pr} f(P-D u S c Q)+\operatorname{Pr} D u S c \gamma \phi\right] \\
& \varphi^{\prime \prime}=\frac{-1}{\operatorname{Pr} S c S r D u-(1+\beta \theta)}\left[\begin{array}{l}
\left.S c S r \beta(P)^{2}+\frac{1}{2} S c f(S r \operatorname{Pr} P-(1+\beta \theta) Q)\right] \\
+(1+\beta \theta) S c \gamma \phi
\end{array}\right]
\end{aligned}
$$

The boundary conditions are

$$
\begin{aligned}
& f(0)=f_{w}, L(0)=0, g(0)=0, \quad \theta(0)=1, \phi(0)=1 \\
& L(\infty)=1, \quad g(\infty)=0, \quad \theta(\infty)=0, \phi(\infty)=0
\end{aligned}
$$

As the initial values for $L(0)=f^{\prime \prime}(0), N(0)=g^{\prime}(0), P(0)=\theta^{\prime}(0)$ and $Q(0)=\phi^{\prime}(0)$ are not specified in the boundary conditions, assume some suitable values for $\mathrm{M}(0), \mathrm{N}(0), \mathrm{P}(0)$ and $\mathrm{Q}(0)$. Then Eqs. (15) are integrated using the fourth order Runge-Kutta method from $\eta=0$ to $\eta=\eta_{\max }$ over successive step lengths 0.01 . Here, $\eta_{\max }$ is the value of $\eta_{\text {at }} \infty$ and chosen large enough so that the solution shows little further change for $\eta$ larger than $\eta_{\max }$. The accuracy of the assumed values for $\mathrm{M}(0), \mathrm{N}(0), \mathrm{P}(0)$ and $\mathrm{Q}(0)$ are then checked by comparing the calculated values of $\mathrm{L}, \mathrm{g}, \theta$ and $\phi$ at $\eta=\eta_{\max }$ with their given value at $\eta=\eta_{\max }$. If a difference exists, another set of initial values for $\mathrm{M}(0), \mathrm{N}(0), \mathrm{P}(0)$ and $\mathrm{Q}(0)$ are assumed and the process is repeated. This process is continued until the agreement between the calculated and the given condition at $\eta=\eta_{\max }$ is within the specified degree of accuracy $10^{-5}$.

\section{Local Skin Friction Coefficient, Surface Temperature and Concentration Distribution}

The skin friction coefficients are denoted by

$$
\begin{aligned}
& \tau_{x}=\mu\left(\frac{\partial u}{\partial y}\right)_{y=0} \text { and } \tau_{z}=\mu\left(\frac{\partial w}{\partial y}\right)_{y=0} \text { which are proportional to } \\
& \left(\frac{\partial^{2} f}{\partial \eta^{2}}\right)_{\eta=0} \text { and }\left(\frac{\partial g}{\partial \eta}\right)_{\eta=0} .
\end{aligned}
$$

The Nusselt number and Sherwood number are denoted by

$$
N u=-\frac{1}{\Delta T}\left(\frac{\partial T}{\partial y}\right)_{y=0} \text { and } S h=-\frac{1}{\Delta C}\left(\frac{\partial C}{\partial y}\right)_{y=0}
$$

In non-dimensional form

$$
N u=\left(\frac{\partial \theta}{\partial \eta}\right)_{\eta=0} \text { and } S h=\left(\frac{\partial \varphi}{\partial \eta}\right)_{\eta=0} .
$$

\section{Comparison with Previous Work and Program Validation}

To validate the numerical method we compare the results with previously published work by Alam and Rahman (2006) these results are reported in Table 1. A comparison of the surface temperature and local skin friction 
coefficient obtained in the present work with $x^{\frac{1}{5}}=\xi, M=0$ and $\gamma=0$ and obtained by Merkin and Pop (1996) and Pozzi and Lupo (1988) have been shown in Table 1 and Table 2, respectively. It is clearly seen that there is an excellent agreement among the respective results.

Table 1: Numerical values of Nusselt number and Sherwood number are compared with those of Alam and Rahman (2006) by taking $\varepsilon=0, \beta=0, \gamma=0, R=0$.

\begin{tabular}{|c|c|c|c|c|c|}
\hline \multicolumn{2}{|c|}{ Parameters } & \multicolumn{2}{c|}{ Alam and Rahman (2006) } & \multicolumn{2}{c|}{ Present Results } \\
\hline $\mathrm{Du}$ & $\mathrm{Sr}$ & $\mathrm{Nu}$ & $\mathrm{Sh}$ & $\mathrm{Nu}$ & $\mathrm{Sh}$ \\
\hline 0.030 & 2.0 & 0.5310 & 0.1292 & 0.5309 & 0.1288 \\
\hline 0.037 & 1.6 & 0.5299 & 0.1605 & 0.5301 & 0.1602 \\
\hline 0.050 & 1.2 & 0.5285 & 0.1921 & 0.5289 & 0.1920 \\
\hline 0.060 & 1.0 & 0.5275 & 0.2077 & 0.5270 & 0.2075 \\
\hline 0.075 & 0.8 & 0.5263 & 0.2233 & 0.5258 & 0.2230 \\
\hline 0.120 & 0.5 & 0.5230 & 0.2470 & 0.5225 & 0.2465 \\
\hline 0.600 & 0.1 & 0.4908 & 0.2817 & 0.4905 & 0.2812 \\
\hline
\end{tabular}

\section{Results and Discussion}

In this study, the efficient method Runge-Kutta method with shooting technique has been used to solve the governing equations (10)-(13) subject to the boundary conditions (14). Selected computations have been found to study the influence of variable porosity regime, variable thermal conductivity and chemical reaction parameter on primary and secondary velocity, skin friction components, Nusselt number and Sherwood numbers. In all cases we have assumed the default values (unless, otherwise specified) for the parameters: $\varepsilon=2$, $\mathrm{R}=0.2, \mathrm{~g}_{\mathrm{s}}=1, \mathrm{~g}_{\mathrm{c}}=0.1, \mathrm{~K}=0.05, \mathrm{Pr}=0.71, \beta=0.5, \mathrm{Sc}=0.22, \gamma=0.5, \mathrm{fw}=0.5$.

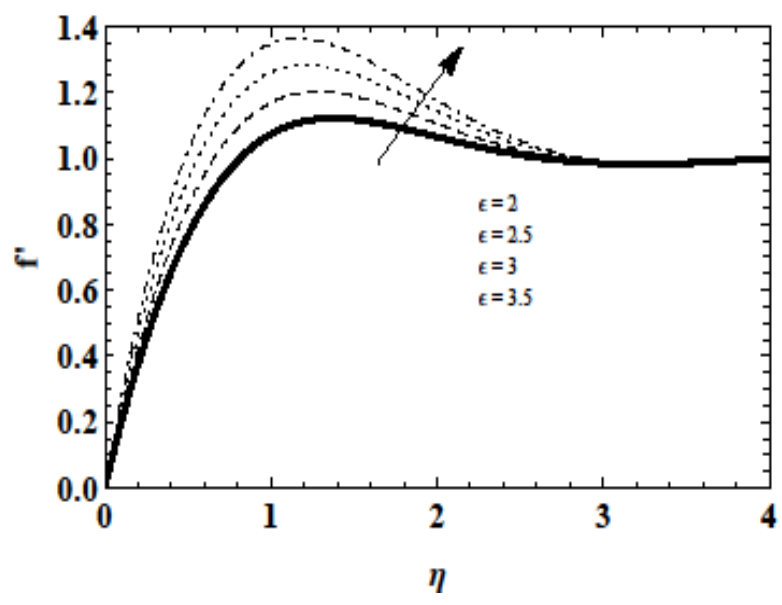

Fig. 2: Velocity profile for various values of variable porosity regime.

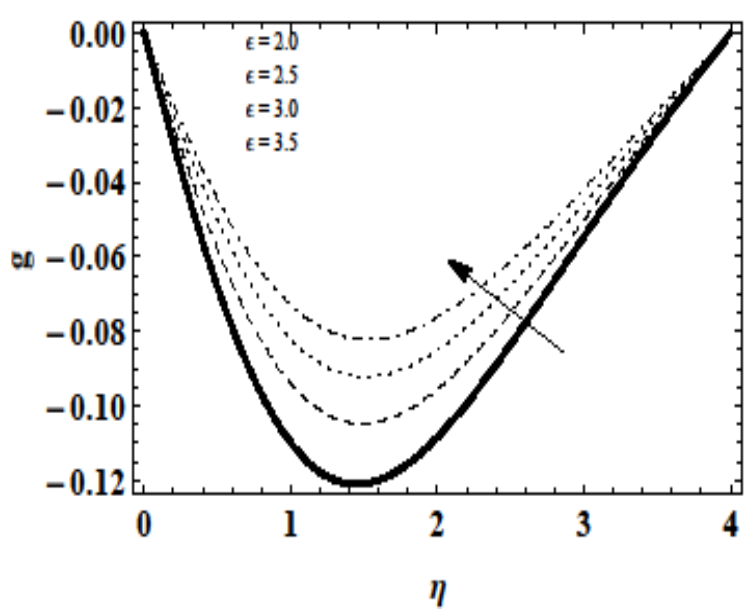

Fig. 3: Secondary Velocity profile for various values of variable porosity regime

Figs. (2) - (5) depicts the influence of porosity $(\varepsilon)$ on the boundary layer variables, primary and secondary velocity profiles, temperature profile and concentration profiles. Increasing porosity results an enhancement in primary and secondary velocity profile but retards in temperature and concentration profiles, i.e. the boundary layer is cooled.

Figs. (6) - (9) illustrates the influence of variable thermal conductivity $(\beta)$ on the boundary layer variables, $f^{\prime}, g, \theta, \phi$. From Fig. (6) We observed that primary velocity increases with increase in $\beta$. i.e., increase the velocity boundary layer thickness. In Fig. (7) a similar response for the secondary velocity field is observed, as with the primary velocity profiles. Secondary velocity values are continuously decreased with increasing 
porosity. An increase in $\beta$, causes an increment in temperature profile with distance into the boundary layer, i.e., accelerate the thermal boundary layer but the concentration profile decreases with increase in $\beta$.

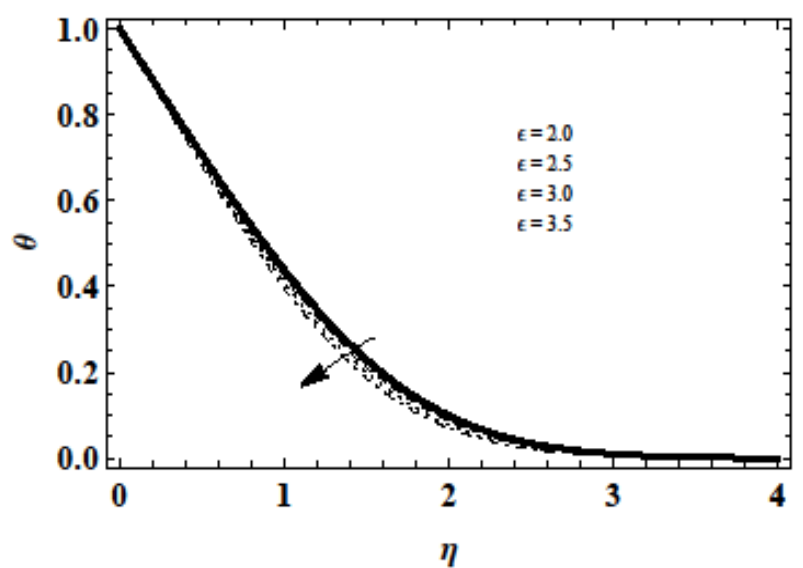

Fig. 4: Temperature profile for various values of variable porosity regime.

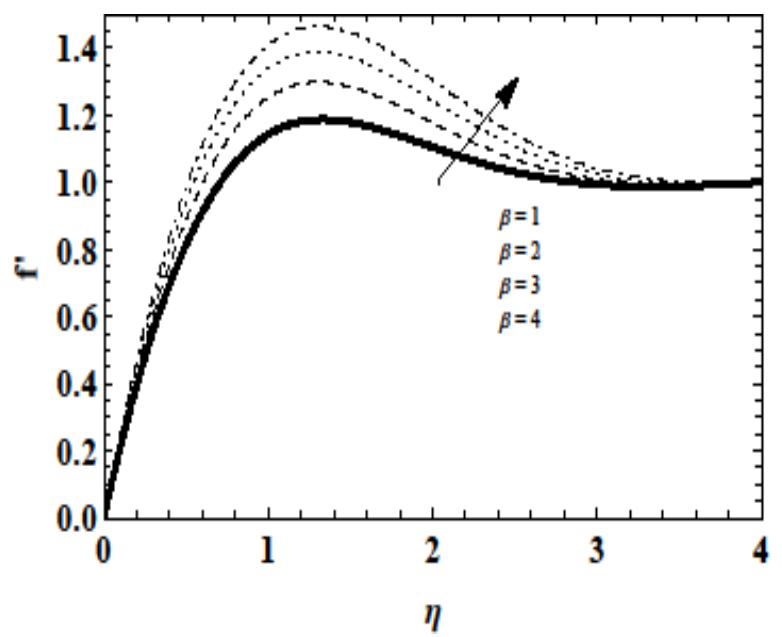

Fig. 6: Velocity profile for various values of variable thermal conductivity.

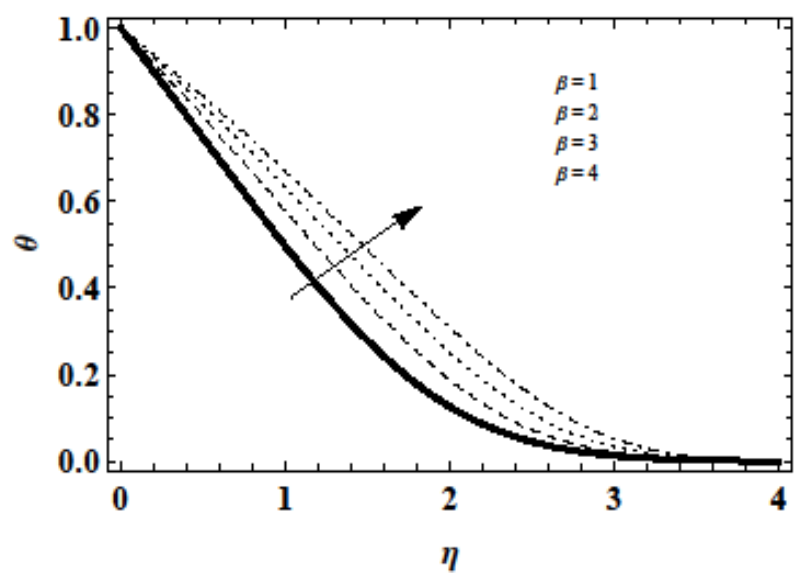

Fig. 8: Temperature profile for various values of variable thermal conductivity.

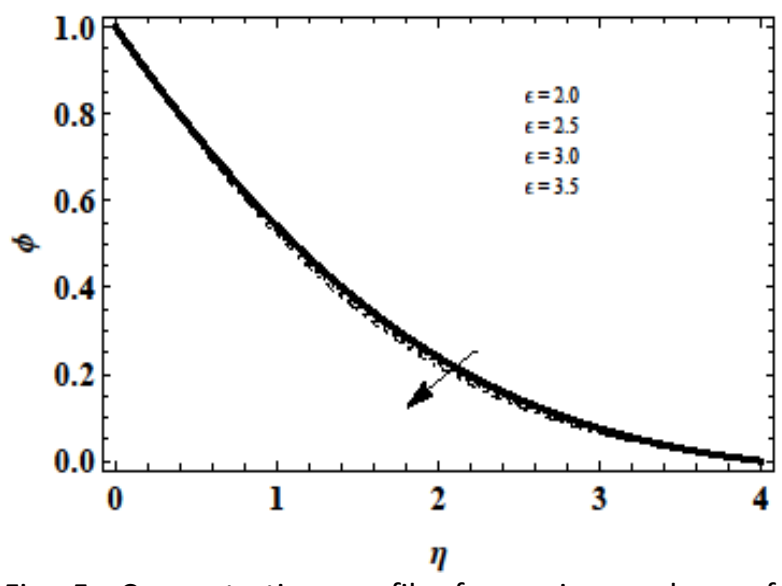

Fig. 5: Concentration profile for various values of variable porosity regime.

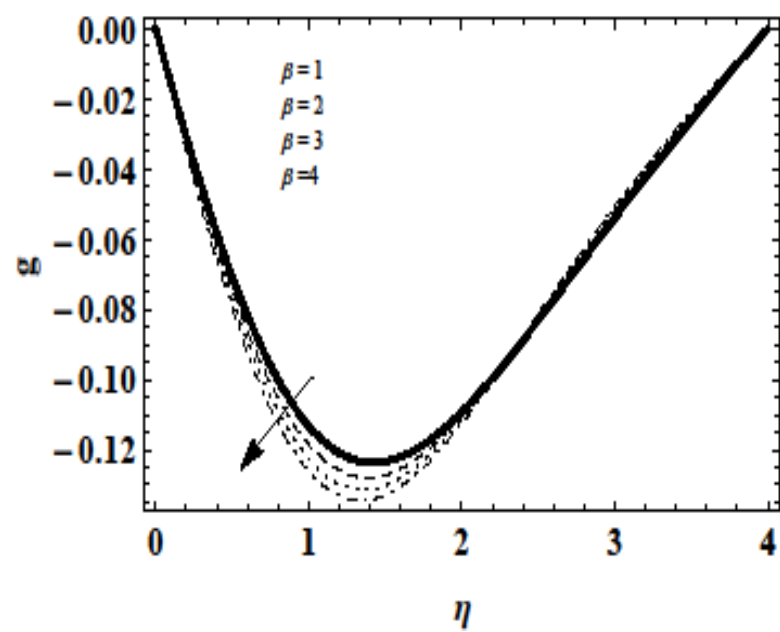

Fig. 7: Secondary velocity profile for various values of variable thermal conductivity

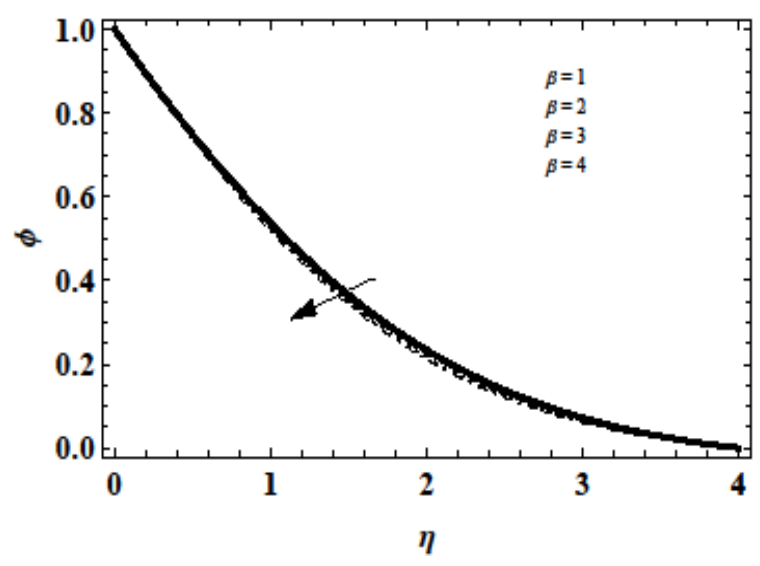

Fig. 9: Concentration profile for various values of variable thermal conductivity. 
Figs. (10) - (11) shows the influence of chemical reaction parameter on primary velocity and concentration profiles. With a dramatic increase in chemical reaction parameter $(\gamma)$ there is a very slight deceleration in primary velocity in the boundary layer regime as shown in Fig. (10). The deceleration in the flow results in thinner velocity boundary layer. It shows that concentration distribution decrease when the chemical reaction parameter is increased and it causes the thinner concentration boundary layer. An increase in concentration of the diffusing species increase the mass diffusion and thus the fluid velocity and temperature increase.

The non-dimensional skin-friction components $\tau_{x}$, and $\tau_{z}$, Nusselt number $(\mathrm{Nu})$ and Sherwood number (Sh) are reported in Tables 2-3 for different values of variable porosity regime $(\varepsilon)$, variable thermal conductivity $(\beta)$ and chemical reaction parameter $(\gamma)$. From Table 2 we observed that increasing $\varepsilon$ tends to accelerate skin friction components $\tau_{x}, \tau_{z}$, Nusselt number and Sherwood number. Increasing $\varepsilon$ clearly serves to reduce presence of matrix fibers in the boundary layer regime which therefore gives rise to a lower resistance to the fluid flow and shear faster past the vertical surface. From Table 3 we say that skin friction component $\tau_{x}$, and Sherwood number increases while skin friction component $\tau_{z}$ and Nusselt number decreases with increase in variable thermal conductivity parameter $(\beta)$ and $\beta=0$ gives the result in case of uniform thermal conductivity. It is obvious that increasing chemical reaction serves to enhance the rate of interfacial mass transfer and hence rate of mass transfer is more pronounced. Thus, from Table 3 it is clear that skin friction component $\tau_{x}$, and Nusselt number decreases while skin friction component $\tau_{z}$ and Sherwood number increases with the increase of chemical reaction parameter.

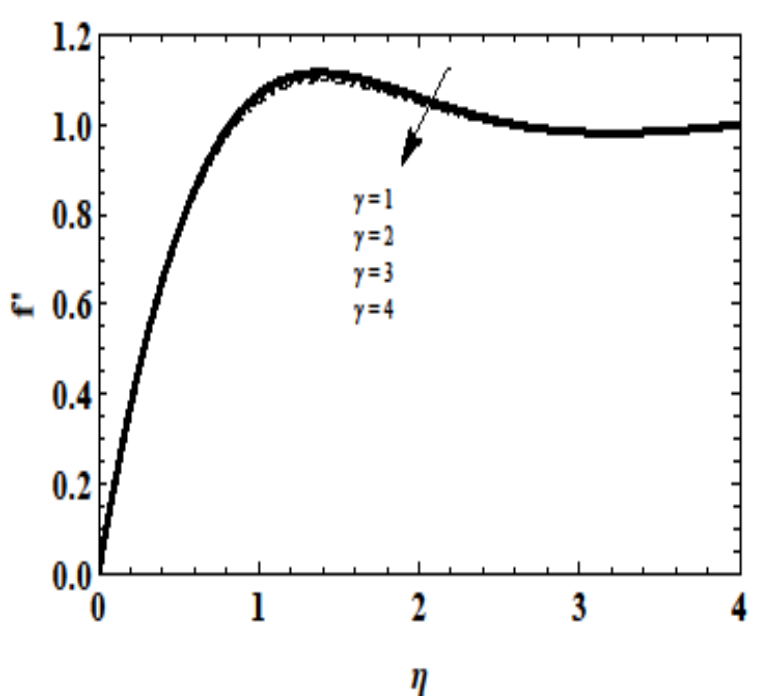

Fig. 10: Velocity profile for various values of chemical reaction parameter.

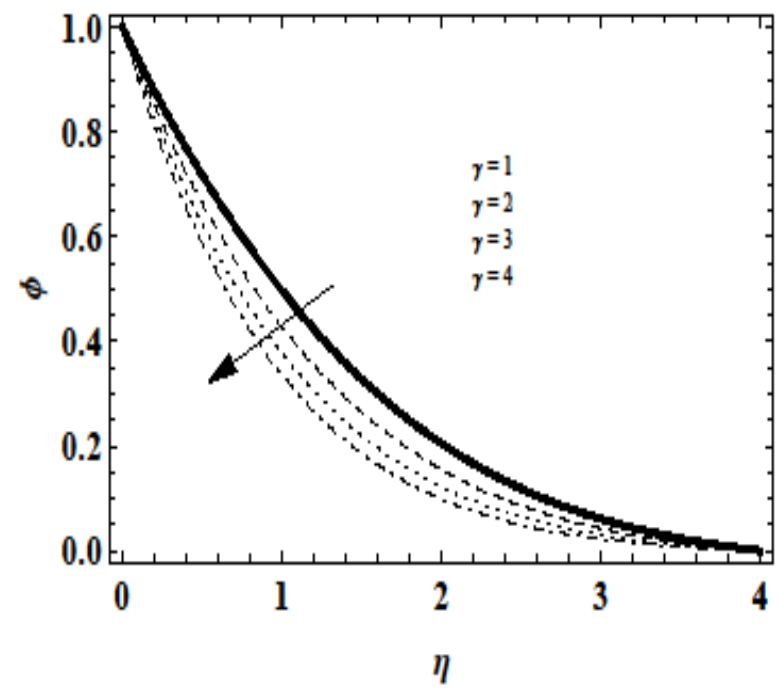

Fig. 11: Concentration profile for various values of chemical reaction parameter.

Table 2: Numerical values of Skin friction components, Nusselt number and Sherwood number for different values of variable porosity regime.

\begin{tabular}{|c|c|c|c|c|}
\hline$\varepsilon$ & $\tau_{x}$ & $\tau_{z}$ & $\mathrm{Nu}$ & $\mathrm{Sh}$ \\
\hline 1.0 & 1.6895 & -0.2698 & 0.5649 & 0.524 \\
\hline 1.5 & 1.8699 & -0.1928 & 0.5787 & 0.531 \\
\hline 2.0 & 2.1375 & -0.1530 & 0.5954 & 0.538 \\
\hline 2.5 & 2.4172 & -0.1282 & 0.6120 & 0.546 \\
\hline
\end{tabular}


Table 3: Numerical values of skin friction components, Nusselt number and Sherwood number for different values of variable thermal conductivity and chemical reaction parameter

\begin{tabular}{|c|c|c|c|c|c|}
\hline$\beta$ & $\gamma$ & $\tau_{x}$ & $\tau_{z}$ & Nu & Sh \\
\hline 1.0 & 0.5 & 2.2454 & -0.1586 & 0.4977 & 0.5435 \\
\hline 1.2 & 0.5 & 2.2826 & -0.1606 & 0.4703 & 0.5454 \\
\hline 1.4 & 0.5 & 2.3171 & -0.1624 & 0.4470 & 0.5471 \\
\hline 1.6 & 0.5 & 2.3494 & -0.1642 & 0.4271 & 0.5487 \\
\hline 0.5 & 1.0 & 2.12901 & -0.1525 & 0.5945 & 0.6293 \\
\hline 0.5 & 1.5 & 2.1220 & -0.1521 & 0.5938 & 0.7106 \\
\hline 0.5 & 2.0 & 2.1159 & -0.1517 & 0.5932 & 0.7847 \\
\hline
\end{tabular}

\section{Conclusion}

From above mentioned studies, following conclusion can be drawn:

- Primary and secondary velocity profiles increases while decrease in temperature and concentration profiles with increase in variable porosity $(\varepsilon)$.

- An increase in variable thermal conductivity cause to increase in primary velocity and temperature profiles while decrease in secondary velocity and concentration profiles.

- Increase in chemical reaction parameter tends to decrease in primary velocity while increase in concentration profile.

- Increasing variable porosity results an enhancement in skin friction components, Nusselt number and Sherwood number.

- Increasing variable thermal conductivity has been shown to elevate skin friction component $\tau_{x}$ and Sherwood number but depress skin friction component $\tau_{z}$ and Sherwood number.

- Increasing chemical reaction parameter have been found to decelerate skin friction component $\tau_{x}$ and Nusselt number and accelerate skin friction component $\tau_{z}$ and Sherwood number.

\section{Acknowledgement}

The authors would like to thank to the Department of Science and Technology, New Delhi, India for providing financial support to enable conducting this research work under Inspire Program.

\section{References}

Alam, M.S. and Rahman, M.M. (2006): Dufour and Soret effects on mixed convection flow past a vertical porous flat plate with variable suction. Nonlinear Analysis: Modelling and Control. Vol. 11, No.1, pp. 3-12.

Al-Humoud, J.M. and Chamkha, A.J. (2006): Double diffusive convection of a rotating fluid over a surface embedded in a thermally stratified high-porosity medium. Heat and Technology, Vol. 24 No. 1 pp. 51-59.

Anwar Beg, O. Takhar, H.S. Beg, T.A. Chamkha, A.J. Nath, G. and Majeed, R. (2005): Modelling convection heat transfer in a rotating fluid in a thermally stratified high-porosity medium: Numerical finite difference solutions. International Journal of Fluid Mechanics Research, Vol. 32 No. 4 pp. 383-401. http://dx.doi.org/10.1615/InterJFluidMechRes.v32.i4.10

Anwar Beg, O. Takhar, H.S. Zueco, J. Sajid, A. and Bhargava, R (2008): Transient couetto flow in a rotating non-Darcian porous medium parallel plate configuration: network simulation method solutions. Acta Meccanica, Vol. 200 pp. 129-144. http://dx.doi.org/10.1007/s00707-008-0040-8

Chauhan, D.S. and Agarwal, R. (2012): Effects of hall current on MHD couette flow in a channel partially filled with a porous medium in a rotating system. Meccanica, Vol. 47, pp. 405-421.

Hassanien, I.A. and Rashed, Z.Z. (2011): Non-Darcy free convection flow over a horizontal cylinder in a saturated porous medium with variable viscosity, thermal conductivity and mass diffusivity. Communications in 
Nonlinear Science and Numerical Simulations, Vol. $16 \quad$ pp. 1931-1941. http://dx.doi.org/10.1016/j.cnsns.2010.08.030

Mbeledogu, I.U. and Ogulu, A. (2007): Heat and mass transfer of an unsteady MHD natural convection flow of a rotating fluid past a vertical flat plate in the presence of radiative heat transfer. International Journal of Heat and Mass Transfer, Vol. 50 pp. 1902-1908. http://dx.doi.org/10.1016/j.ijheatmasstransfer.2006.10.016

Mukhopadhyay, S. (2009): Unsteady boundary layer flow and heat transfer past a porous stretching sheet in presence of variable viscosity and thermal diffusivity. International Journal of Heat and Mass Transfer, Vol. 52, pp. 5213-5217. http://dx.doi.org/10.1016/j.ijheatmasstransfer.2009.04.013

Narayana, M. Khidir, A.A. Sibanda, P. and Murthy, P.V.S.N. (2013): Viscous dissipation and thermal radiation effects on mixed convection from a vertical plate in a Non-Darcy porous medium. Transport in Porous Media, Vol.96, pp.419-428. http://dx.doi.org/10.1007/s11242-012-0096-8

Pal, D. and Mondal, H. (2009): Radiation effects on combined convection over a vertical flat plate embedded in a porous medium of variable porosity. Acta Meccanica, Vol. 44, pp. 133-144.

Pal, D. (2010): Magnetohydrodynamic non-Darcy mixed convection heat transfer from a vertical heated plate embedded in a porous medium with variable porosity. Communication in Nonlinear Science and Numerical Simulation, Vol. 15, pp. 3974-3987. http://dx.doi.org/10.1016/j.cnsns.2010.02.003

Prasad, V.R. Vasu, B. Anwar Beg, O.and Parshad, R.D. (2012): Thermal radiation effects on magnetohydrodynamic free convection heat and mass transfer from a sphere in a variable porosity regime. Communication in Nonlinear Science and Numerical Simulation, Vol. 17 pp. 654-671. http://dx.doi.org/ 10.1016/j.cnsns.2011.04.033

Salah, F. Aziz, Z.A. and Ching, D.L.C. (2011): New exact solutions for MHD transient rotating flow of a second-grade fluid in a porous medium. Journal of Applied Mathematics, pp. 1-8.

Schwartz, C.E. and Smith, J. M. (1953). Flow distribution in packed beds. Ind Eng Chem, Vol. 45, pp. 12091218.

Seddeek, M.A. and Salama, F.A. (2007): The effects of temperature dependent viscosity and thermal conductivity on unsteady MHD convective heat transfer past a semi-infinite vertical porous moving plate with variable suction. Computational Material Science, Vol. 40 pp. 186-192. http://dx.doi.org/10.1016/ j.commatsci.2006.11.012.

Srinivasacharya, D. and Swamy Reddy, G. (2013): Mixed convection heat and mass transfer over a vertical plate in a power-law fluid-saturated porous medium with radiation and chemical reaction effects. Heat TransferAsian Research, Vol.42(6), pp. 485-499. http://dx.doi.org/10.1002/htj.21058

Vafai, K. and Tien, C.L. (1981): Boundary and inertia effects on flow and heat transfer in porous media. International Journal of Heat and Mass Transfer, Vol. 24, pp. 195-203. http://dx.doi.org/10.1016/0017$\underline{9310(81) 90027-2}$

Vajravelu, K. Prasad, K.V. and Chiu-on, Ng. (2013): Unsteady convective boundary layer flow of a viscous fluid at a vertical surface with variable fluid properties. Nonlinear Analysis: Real World Application, Vol. 14, pp. 455-464. http://dx.doi.org/10.1016/j.nonrwa.2012.07.008 